\title{
LA IDENTIFICACIÓN DE TALENTOS DEPORTIVOS PARA DEPORTES DE HABILIDAD ABIERTA. UNA VISIÓN SOCIO DEPORTIVA
}

\author{
THE IDENTIFICATION OF SPORTS TALENTS FOR OPENED SKILL SPORTS. \\ A SOCIAL SPORT VISION
}

Rubén Ricardo Capetillo Velásquez ${ }^{1}$

\section{Resumen}

\begin{abstract}
El presente trabajo analiza algunos estudios que instan a reflexionar sobre los factores que intervienen en el proceso de detección de talentos deportivos desde una óptica social-deportiva. Se pretende responder a inquietudes relacionadas con las capacidades deportivas potenciales, identificación de superdotación sicomotriz, desarrollo del talento y experticia deportiva. Para un desarrollo formativo y posteriormente altos niveles de rendimiento deportivo es esencial contar con estudios, investigaciones y pautas para detectar niveles de superdotación deportiva en niños, proceso que actualmente no existe en nuestra región. Al parecer seguimos aún creyendo que la mejor escuela es la calle y se continúa pensando que el talento deportivo nace y no se hace.
\end{abstract}

A modo de conclusión se recomienda que un programa de identificación de superdotación sicomotriz se debe realizar alrededor de los nueve y diez años, tomando como referente el desarrollo general del niño y no la performance deportiva, ni rígidas mediciones antropométricas. En definitiva, el propósito de este artículo es analizar la superdotación sicomotriz en la fase inicial del proceso de detección de talento deportivo.

Palabras claves: proceso, identificación, talentos, detección, deporte.

\section{Abstract}

The present work analyses some studies that urge to reflect on the factors that intervene in the process of detecting sports talents from a social-sports point of view. It is intended to answer the queries related to the potential sports capacities, the identification of psychomotor giftedness, and the development of talent and sports expertise. In order to foster a formative development, and subsequently, high levels of sports performance, it is fundamental to have studies, investigations and criteria to detect sports giftedness levels in children, a process that currently does not exist in our region. It all seems to indicate that we continue to believe that 'the best school is the street', and it is even still thought that a sports talent is born not made.

As a conclusion, it is recommended that a psychomotor giftedness identification program should take place when the child is around nine or ten years, taking as a reference the general development of the child instead of their sports performance or rigid anthropometric measurements. To summarize, the purpose of this article is to analyze the psychomotor giftedness in the initial phase of the identification process of sports talent.

Key words: process, identification, talents, detection, sports.

Fecha de recepción: 18 de septiembre de 2010

Fecha de aprobación: 26 de octubre de 2010

\footnotetext{
${ }^{1}$ Doctor en Educación Física y Deporte, Universitat de Valencia, España. Magíster en Educación, Mención administración educacional, Universidad de Tarapacá, Arica. Licenciado en Ciencias de la Actividad Física y del Deporte Ministerio de Educación y Ciencia, Madrid, España. Profesor de Educación Física, Universidad de Tarapacá, Arica. Académico Departamento de Educación y Humanidades, Universidad de Tarapacá, Arica, Chile.rucave@alumni.uv.es
}

Universidad Pedagógica Nacional 


\section{Introducción}

¿Por qué nuestros niños con potencialidades deportivas en nuestra región no alcanzan la categoría de experto en alguna disciplina deportiva olímpica? Shonborn (1984) nos orienta al respecto cuando explica que es frecuente realizar el proceso al revés, "buscamos niños que jueguen, lo hacemos participar en competencias y finalmente intentamos saber si tiene talento para el deporte, entonces se produce el fracaso".

Hoy en día la mayoría de los países sudamericanos sustenta y financia al joven deportista de elite, tomando como criterio único los resultados, en una etapa tardía, cuando éste ha obtenido algunos rendimientos significativos a nivel nacional, en gran medida debido al esfuerzo y sacrificio personal-familiar, con todas las carencias que esto significa.

Contar con deportistas excelentes requiere de un sistemático proceso de detección de talentos, el cuál se torna aún más complejo cuando la elección es un deporte "sociomotriz" (Parlebas, 1997; Hernández y Jiménez, 2000). Un proyecto significa considerar entre otros factores, programas específicos de búsqueda, equipo multidisciplinario, técnicos deportivos especializados, planificación de un alto nivel de competencias periódicas y fundamentalmente del apoyo financiero en el momento adecuado, "el acceso a la élite deportiva no es producto de la suerte ni del azar, y mucho menos de la predestinación" (Rius i Sant, 1995).

Es esencial contar con estudios, investigaciones y pautas para detectar niveles de superdotación deportiva en niños, proceso que actualmente no existe en nuestra región. Parafraseando a Campos (1996), al parecer seguimos aún creyendo que "la mejor escuela es la calle" y se continúa pensando que el talento deportivo nace y no se hace.

Lo incierto del futuro deportivo de un niño poseedor de talento motriz en ciertas ocasiones frena el ímpetu familiar o siembra dudas en las instituciones patrocinantes en cuanto a inversión económica debido a las múltiples variables que influyen en la etapa de desarrollo y posterior concreción. Lo lógico y conveniente sería lograr convencer a las autoridades competentes, que éstos entiendan que optar por un proceso sistemático de búsqueda de talentos significaría, de acuerdo a Bompa (1987), que los resultados que se pudiesen obtener servirían para no provocar inversiones innecesarias en clubes e instituciones y falsas esperanzas en las familias. La "captación" (1997) de un talento deportivo es una tarea difícil pero necesaria para el futuro de los clubes.

En los últimos veinte años la competición deportiva de la primera región chilena evidencia resultados negativos en el deporte de alto nivel de competición. Ausencia de figuras mundiales y referentes deportivos, carencia de triunfos internacionales, mínima contribución de seleccionados para Chile en deportes competitivos y profesionales de repercusión mundial. Los deportistas seleccionados que se encuentran actualmente inmersos en competencias internacionales no alcanzan destacada figuración.

La decisión federativa o de asociaciones deportivas aún mantiene un grado de incredulidad a la hora de tomar la decisión de abordar un proceso de detección de talentos deportivos. Es posible pensar que entre las principales vacilaciones que les impide decidirse es la inseguridad de si: ¿es posible identificar niños con dotación deportiva a temprana edad?, ¿existen especialistas e instrumentos confiables necesarios para el proceso de detección del talento en su fase inicial? y ¿en que época evolutiva y según que patrones se ha de identificar al niño con talento?

\section{El proceso de detección del talento}

Todavía no existe consenso entre investigadores en cuanto al término correcto a recurrir cuando se trata de buscar y hallar un posible talento deportivo. Los conceptos empleados para representar el proceso se confunden en una nebulosa terminológica: identificación, selección, búsqueda, detección, captación, son algunos de los vocablos que más que aclarar y definir, enmarañan el proyecto de itinerario deportivo que habrá de seguir un niño con súperdotación deportiva.

Sin embargo, algunos estudios sugieren patrones que contribuyen a consensuar lineamientos para estructurar etapas y secuencias lógicas de acciones operacionales en el tiempo.

Campos (1996), dice que el "proceso de detección de talentos" es una organización sistemática estructurada por fases y objetivos concretos, tendientes a 
confirmar el talento al final del itinerario deportivo, catalogando a éste como exitoso si es que sitúa al deportista a las puertas del alto nivel de rendimiento deportivo.

Leger y Cloutier en Dosil (2001), determinan que la Identificación de un talento deportivo consiste en predecir si ese joven deportista podrá desarrollar el potencial de adaptación al entrenamiento y su capacidad de aprendizaje técnico, saber si a largo plazo podrá alcanzar altos rendimientos.

Salmela y Régnier (1983) lo entienden como una forma de predicción en el tiempo en cuanto a las posibilidades de que un deportista posea las capacidades necesarias para alcanzar un nivel de rendimiento dado en un deporte específico.

Para el Director técnico de la federación Checoslovaca de tenis Frantisek Slezak, la identificación de un talento es un proceso más o menos continuo y a largo plazo y sus bases estarían establecidas por los propios deportes.

Torres (1998) especifica que la detección precoz hay que entenderla como descubrir, dar a conocer posibilidades que no se conocen pero que están en el individuo.

Estas definiciones estarían indicando que el tiempo es una variable clave para la elaboración de un programa manteniendo implícita la sensación de predicción e incertidumbre en cuanto a éxito.

Por lo tanto podríamos pensar en primera instancia que la intención de un programa consistiría en idear un sistema destinado a evaluar una población concreta de niños de tal manera de identificar a los más competentes y promisorios. Ésta es una cuestión que requiere de reflexión, estudio y de una rigurosa planificación estratégica para no excluir del programa a ningún chico con condiciones.

Un proceso de detección de talentos comienza con la "identificación" (García, et al., 2003), ésta representaría el primer paso para seleccionar niños con las aptitudes necesarias para conseguir en los siguientes años los más altos niveles de perfomance deportiva a través de un complejo proceso de especialización. Es necesario dejar claro que esto no asegura el éxito futuro, es tan sólo un primer paso, los verdaderos rendimientos deberían darse varios años después.
La Identificación es precoz, corresponde realizarla durante la infancia, considerando el desarrollo global del niño más que el conseguir un registro deportivo extraordinario. Fundamental es considerar que cada seleccionado goce de un estado saludable, Bompa (1987) lo dice así: de nada sirve tener un talento si no tiene salud.

Por su parte, Dosil (2001) señala que adoptar una postura reduccionista en la detección del talento basada exclusivamente en las cualidades físicas no es lo óptimo, es fundamental tener en cuenta también las variables sicológicas y sociales. Agrega además que la edad óptima para una primera selección es coincidente con la etapa escolar y varía según la modalidad deportiva, a pesar de las semejanzas estructurales que se pudiera encontrar entre familias de deportes.

Es el ámbito escolar por excelencia el lugar más idóneo para comenzar la etapa de identificación, por ser éste un entorno donde es posible asegurar la participación del chiquillo en actividades deportivas durante varios años. En este medio social es probable descubrir y percibir potencialidades que no se conocen pero que están en el niño, lo que permitiría realizar en el tiempo un seguimiento y control para valorar la evolución de esas capacidades. Autores como Gutiérrez y García Ferrol (2001) consideran también al contexto escolar como poseedor de gran poder de influencia y supone un importante referente para los niños, donde el profesor de educación física emerge como el adulto significativo más importante.

Es conveniente señalar que las clases de Educación Física contempladas y estructuradas en los programas del ministerio de educación permiten al alumno desarrollar las habilidades motoras básicas necesarias que posibilitarían en una instancia inicial, identificar y seleccionar niños con superdotación deportiva. Resulta por cierto importante que el profesor de educación física posea también algunos recursos adicionales, perfeccionamiento continuo y se le destinen horas para trabajar en la gestión de programas específicos de identificación, sin esto no sería posible seleccionar adecuadamente a los mejores. Un medio ambiente escolar de este tipo se convierte en un periodo vital donde se produciría el primer momento o fase de la detección de talentos deportivos. 
Es aquí donde el proyecto educativo juega un papel clave, cada escuela tendría que incluir horas necesarias y quizás adicionales de actividad física e iniciación deportiva sistemática en el primer ciclo básico de enseñanza. Estos años representan instancias decisivas, constituyéndose en el marco más adecuado para desarrollar las fases iniciales de cualquier programa deportivo (Campos, 1996). En este periodo es conveniente mantener una relación permanente y coordinada entre deporte de competición escolar y deporte de asociación.

$\mathrm{Al}$ parecer existiría cierto encuentro en cuanto a una primera fase inicial de búsqueda denominada "identificación" de la superdotación deportiva, como también el largo plazo en el tiempo para que el niño superdotado deportivamente se desarrolle a plenitud.

En consecuencia, efectuar una primera selección se convierte en el proceso a través del cual se identifican las habilidades, destrezas y capacidades sicomotrices, disposición psicológica y factores sociales que constituirían condiciones necesarias para asimilar cargas de entrenamiento futuras, afines a los objetivos correspondientes según la etapa de formación en que se encuentre el deportista.

Las investigaciones proponen un plan sistemático de muy largo plazo para desarrollar "la potencialidad deportiva latente", donde se incluye además un periodo de seguimiento para que al cabo de unos diez años de formación, recién confirmar la condición alcanzada de "experto" (Lorenzo, 2001) y posteriormente llegar a "la cúspide de la maestría" (Platonov, 1995) en cuanto rendimiento deportivo.

Un proyecto de este tipo debería aspirar a seleccionar deportistas de excelencia deportiva, que luego puedan ser reconocidos internacionalmente y además se conviertan en referentes de generaciones venideras, de acuerdo a Marco (2003) seleccionar al atleta con facultades físicas, preparación técnica y características sicológicas únicas y especiales. El propósito fundamental es "ayudar sólo a los jugadores con posibilidades reales" (Stojan, 1984).

\section{Superdotación y talento deportivo}

La identificación de niños con ciertas habilidades sicomotrices, la mayor de las veces se efectúa de manera superficial, generalmente basado en la subjetividad del observador, selección que carece de fundamentos teóricos y científicos de respaldo. Actualmente identificar un superdotado deportivo requiere de algo más que el conocimiento intuitivo del "veedor".

Los programas de selección de talentos deportivos generalmente se inician con mucho entusiasmo y esperanzas efectuándose principalmente en competencias deportivas importantes de series menores, lamentablemente los campeonatos deportivos no son el lugar indicado para su detección. Un técnico especialista como el alemán Shonborn manifiesta que este método de búsqueda no sólo es inútil, sino que va en dirección contraria a lo que científicamente debería ser un proyecto de este tipo.

Tal como acontece con las palabras empleadas para definir el proceso de detección de talentos, aún no ha sido posible un acuerdo en los términos empleados para definir a estos tipos de niños. Los vocablos provienen de los países anglosajones: gifted (superdotado) y talent (talento). Muchos autores le asignan significados semánticos parecidos y es Gagné quien traza diferencias al definir la superdotación como capacidad natural o "desarrollo asistemático" de las habilidades humanas en forma de potencialidad latente, mientras que el talento lo concibe como el desarrollo sistemático de las habilidades que concede el nivel de experto en algún campo del comportamiento humano.

La "superdotación deportiva" compete al ámbito de la motricidad, por lo tanto hablamos de una superioridad sicomotriz que sitúan al niño más allá de la generalidad de los compañeros de su edad biológica (Terreros, 2002), independiente del deporte que practique. Es decir, deportistas infantes que poseen características motrices sobre el promedio, no necesariamente muy superiores pero que ya dejan ver una potencialidad latente, que sólo alcanzará la categoría de experto cuando se dedique a perfeccionarlas.

En cuanto a talento, la diferencia la aclara Dosil (2001), cuando dice que el niño con "talento deportivo" posee una habilidad especial que les permite ser más competentes que la mayoría de la población en un deporte específico. Son niños que nacen con características especiales que le permiten, median- 
te un eficaz entrenamiento y adecuadas condiciones ambientales, optar a ser campeones en un determinado deporte.

Pila, en Moraes (2005), especifica que el talento en el ser humano, es toda conducta supranormal o sobresaliente en las manifestaciones de este como ser social, ya sea en el arte, la cultura, las ciencias o el deporte. Hann (1988) define el talento deportivo como la disposición por encima de lo normal de poder y querer realizar unos rendimientos elevados en el campo del deporte, lo que le obliga a someterse a un programa de entrenamiento deportivo para conseguir éxitos y rendimientos extraordinarios.

Por su parte, Vergara (1999) encuentra que los niños dotados y talentosos son aquellos identificados por personas profesionales calificadas, quienes por virtud de habilidades destacadas, son capaces de un alto rendimiento. Seirullo (2000), considera que son aquellos individuos que hacen cosas imprevisibles porque están en un punto de desequilibrio y precisamente por no haber sido sometidos a los procesos lineales con los que se entrena no se le ha destruido esa capacidad para responder como un sistema abierto, no lineal.

Shonborn (1984), determina al talento como "la evolución progresiva hasta la plasmación definitiva de una aptitud excepcional, es decir, la aptitud es un requisito constante que se tiene o no se tiene". Podemos caracterizar entonces al talento deportivo como: al adolescente que posee competencias condicionales y deportivas específicas por encima de los patrones normales, sustentadas en la posibilidad futura de que éstas alcancen un alto nivel de perfeccionamiento, rendimiento y resultado.

\section{Condiciones socio deportivas para su desarrollo}

Hablar de formación eficaz del superdotado sicomotor y posterior especialización del talento deportivo, es decir, ambiente social estimulante que rodea al sujeto, puesto que de nos ser así, podría no desarrollarse o hacerlo mínimamente (Dosil, 2001).

La situación geográfica y el contexto social en el rendimiento deportivo se plantean fundamentales para la mejora del deportista, lo cual implica examinar, considerar y controlar el área geográfica de residencia del teórico talento deportivo. Ésta variable podría o no, favorecer la iniciación y promoción de jugadores en un tipo de deporte concreto. Capetillo (2008), determina que los deportistas sometidos a intensas y constantes sesiones de entrenamiento, deben poseer condiciones de vida con un medio ambiente y entorno geográfico favorable. También es decisivo el nivel de competición en el que se desarrollan los talentos, así como el clima que sea capaz de gestionar el entrenador (Sáenz-López et al., 2003).

Un factor significativo para un progreso óptimo es el rol que debe desempeñar la familia, ésta proporciona el ambiente social primario donde el deportista puede desarrollar su identidad, autonomía y motivación para lograr el éxito (Gutiérrez y García Ferrol, 2001). El papel de los padres posee evidentemente un peso elemental en los logros deportivos de sus hijos. Las motivaciones esenciales para que el niño se acerque a un deporte específico las crean éstos a través de organización, control de evolución del rendimiento, premios, apoyo económico y moral (Fuentes, et al., 2003; Hann, 1988). También resulta fundamental en los primeros años contar con una atención médica periódica paralelo al apoyo familiar constante.

Un buen ejemplo lo encontramos en Ruiz Pérez (1999), quien al entrevistar a nadadores y tenistas considerados desde la infancia como talentos, manifestaron que lo que realmente les llevó a ser notables en su ámbito fue su compromiso y deseo de ser excelente, en un entorno familiar favorable y con un entrenamiento adecuado para el desarrollo de sus aptitudes y cualidades. Marco (2003), apunta que sólo se puede llegar a ser el mejor del mundo dedicándose por entero a ello, dice que el deportista tendrá que ser capaz de soportar una férrea disciplina, entrenamientos profesionales duros y limitaciones en sus hábitos de vida.

En cuanto a oferta motriz Hann (1988) propone que en la fase inicial ésta debe ser amplia y variada. Cuántas más opciones de realizar movimientos existan, mayor enfoque para seleccionar y enseñar en las etapas siguientes.

Otras variables a contemplar relacionadas directamente con las características del niño han de ser: destreza, estabilidad psíquica, motivación para el deporte, condición morfológica y atlética respaldada con aspectos hereditarios. Podríamos agregar tam- 
bién, capacidad de aprendizaje en el área motora, resistencia mental, autoconfianza, concentración. Importante y conveniente además es el evaluar la determinación, es decir, el deseo de ser mejor que los otros y las ganas de aprender. Algunos otros factores influyentes que intervienen en el proceso serían el rendimiento escolar, nivel socio económico, nivel del club deportivo, calidad y cantidad de infraestructura, material deportivo, tecnología, ayuda institucional.

Como en todo programa formativo, existe una fase que está fuertemente relacionada con el momento de la iniciación deportiva. Platonov (1995), encuentra que la edad de iniciación en la práctica deportiva ejerce una determinada influencia en la duración de la preparación durante la carrera del deportista.

Por otra parte, entre las dificultades que pudiesen retardar, limitar o simplemente hacer fracasar el desarrollo deportivo, es el grado de especialidad profesional de quien asume la tarea de formador. El niño sobredotado motrizmente requiere de un elaborado programa racional estructurado en fases de desarrollo, lo que significa solicitar un experto que cumpla sistematizar programas de entrenamiento con fundamento científico y pedagógico, no perdiendo ninguna vez la perspectiva de que pocos años más tarde habrá que seleccionar a los mejores para determinados deportes.

En todo caso, cualquiera sea la etapa del proceso y características genéticas, resulta fundamental que el joven durante se itinerario deportivo precise de condiciones socio-ambientales y apoyos técnicodeportivo especiales.

Como se aprecia, no resulta fácil la tarea a la hora de efectuar la elección correcta de los niños con potencialidades deportivas, son muchísimas las variables por atender para luego aventurar una predicción de éxito en el futuro. Se arriesgan cientos de horas de formación, uso de instalaciones, recursos humanos especializados, giras, competencias y todo lo que conlleva formar deportivamente un ser humano.

Si se hace mal, ¿cuánto dinero se habrá perdido?, pero y si no se hace, deportivamente ¿cuántos niños habremos perdido?

Lúdica pedaqóq. Vol 2, No. 15 (ene- dic. 2010) pp. 148 - 155
Al parecer "el campeón nace y después se hace", nacer significaría poseer todas las cualidades vitales para sobresalir en el deporte (genética), y el se hace simbolizaría entrenar al máximo en óptimas condiciones medio ambientales.

\section{Baterías de test}

La batería de test tiene la propiedad de entregar datos cuantitativos que permiten acercarnos a la identificación de niño superdotado, cifras que indicarían posibles potencialidades de rendimiento y adaptación al entrenamiento o capacidad de aprendizaje técnico.

Esta nos provee de información útil y pertinente del niño y los datos obtenidos ya no provienen del azar u "ojo clínico", sino que responden a una metodología y condicionantes del criterio científico.

Una aplicación bajo estrictos protocolos hace que las mediciones de la condición física sirvan para una mejor toma de decisión en cuanto a identificación y calificación de los niños de un grupo escolar o club específico, clasificándolos en niveles de mayor o menor capacidad o rendimiento físico en un momento determinado. Moraes (2005) manifiesta que los test de control físico también se utilizan para diagnosticar el nivel del rendimiento del posible talento y constituyen un acicate de su interés por el entrenamiento.

La batería de test tiene la propiedad de entregar datos cuantitativos que permiten acercarnos a la identificación de niño superdotado, cifras que indicarían posibles potencialidades de rendimiento y adaptación al entrenamiento o capacidad de aprendizaje técnico. Es posible constatar a medida que se estudian las diferentes baterías, que existen "pruebas básicas comunes", luego hay diferencias en el número de estas pruebas y se difiere en la tecnología a utilizar según el país, según la especialidad deportiva y el nivel de perfeccionamiento del deportista.

La estructuración y confección de una batería de test para identificar niños con sobredotación deportiva se debería basar en componentes que sean pertinentes e influyan en el rendimiento y por lo tanto sean estos susceptibles de valorar. Hann (1988), propone que los factores que intervienen sobre el ta- 
lento deportivo serían: requisitos antropométricos, características físicas, condiciones tecno-motrices, capacidad de aprendizaje, dirección cognoscitiva, factores afectivos y condiciones sociales.

Richard Shonborn (1984), sugiere que es conveniente buscar el talento alrededor de los 10-11 años, un talento general para todos los deportes. Indica además que se deben seguir estrategias de selección basadas en patrimonio genético (cualidades innatas), facilidad para aprender y aptitudes cinéticas y deportivas.

Stojan, (1984) por su parte propone en principio realizar una pre selección mediante test simples a niños de entre 7 y 10 años para el caso del tenis y considera que esta búsqueda de talentos no debe limitarse e clubes sino también en colegios y centros deportivos. Entre las característica a encontrar nombra las condiciones atléticas, destreza, agilidad, rapidez, control y habilidad de controlar un balón de fútbol, baloncesto, etc.

De acuerdo a nuestros estudios, como sugerencia se recomienda que un programa de identificación de superdotación sicomotriz deba realizarse alrededor de los nueve y diez años, tomando como referente el desarrollo general del niño y no la performance deportiva, ni rígidas mediciones antropométricas.

\section{Condiciones regionales}

¿Qué condiciones tiene nuestra región para desarrollar la superdotación y talento deportivo? 30 años atrás la ciudad proporcionaba la oportunidad de jugar libre y espontáneamente al contar con amplias calles y un mínimo tráfico vehicular por lo que se hacía habitual "armar pichangas" callejeras durante horas. Hoy, la modernización y explosión demográfica de la ciudad quitó de en medio los espacios deportivos naturales.

No hace muchos años también las amplias y cercanas playas motivaban a realizar gran cantidad y variada actividad deportiva, actualmente ya no es posible gracias al privilegio que se le otorga al turismo, lo que limita y prácticamente prohíbe juegos con balón y paletas, por nombrar algunos.
Una condición vital para el desarrollo del superdotado deportivo es poseer este valioso contexto, un mundo de estímulos, sociales, motores y cognitivos donde sea posible vivenciar y disfrutar en libertad lo que brinda el medio ambiente.

Heredar la tendencia natural por el deporte, poseer el espacio físico, amigos de juego, familia con aficiones deportivas, profesores amante de los deportes, clubes deportivos, modelos de ídolos deportivos, nutrición ligada al mar y por sobre todo un estado saludable. Todas éstas variables entremezcladas sin duda se palpaban en nuestra región.

Este escenario permitía ejercitar en forma natural lo que hoy artificialmente se aspira a lograr a través de las llamadas escuelas de fútbol. La cantidad de horas disponibles en esos años daban oportunidad para realizar intuitivamente casi sin límite las fases de percepción, decisión y ejecución motriz, la toma de decisiones instantáneas en relación a los compañeros y oponentes e ir perfeccionando la acción técnica-táctica y experimentar estrategias ante tan disímiles y heterogéneos contrincantes, trascendentales para el futuro táctico-intelectual del talento deportivo.

Lo anterior nos demuestra que la pronta estereotipación y estandarización de querer "hacer a la medida" lo más pronto posible y que el niño se parezca a un "jugador adulto" en las etapas de iniciación no sólo es poco pedagógico sino limitante para el rendimiento posterior.

Hoy es absolutamente necesaria la tecnología deportiva, darle al trabajo de equipo la importancia que se merece y escalar a lo más alto del conocimiento aplicando practicidad a nuestro trabajo, sólo así, optimizaremos los recursos escasos pero que son propios... nuestros niños.

\section{Referencias}

Año, V. (1997). Planificación y organización del entrenamiento Juvenil, Madrid, Editorial Gymnos.

Bompa, T. (1987). 'La selección de atletas con talento'. En Revista de entrenamiento deportivo, Volumen I, № 2.

Campos, J. (1996). Análisis de los determinantes sociales que intervienen en el proceso de detección de talentos en el deporte, Madrid, Investigaciones en Ciencias del Deporte, ICD, № 3, Ministerio de Educación y Ciencia, Consejo Superior de Deportes, 7-68. 
Capetillo, R. (2008). Análisis de los Factores Socio-Deportivos y Ambientales que Intervienen en la Trayectoria de Futbolistas Juveniles, Argentina, PubliCE Premium.

Dosil, J. (2001). Psicología y deporte de iniciación, Detección de Talentos deportivos, Ediciones Gersam, Ourense, 79-92.

García, J., Campos, J., Lizaur, P., Pablo, C. (2003). El talento deportivo, Madrid, Gymnos.

Gutierrez, M., García, A. (2001). Psicología y deporte de iniciación. El entorno escolar-familiar y la práctica deportiva en la adolescencia: una aproximación empírica, Ediciones Gersam, Ourense, 35-53.

Hahn (1988). Entrenamiento con niños, Barcelona, Martínez Roca.

Hernández, J., Jiménez, F. (2000). 'Los contenidos deportivos en la educación física escolar desde la praxiología motriz'. Obtenido, desde: http://www.efdeportes. com/efd19a/prax1.htm

Lorenzo, A., Refoyo, I., Sanpedro, J., Mundina, J., Gonzalez, I. (2001). Análisis comparativo de las acciones realizadas en competición, entre jugadores expertos y novatos, en baloncesto, en categorías de formación. Nuevas aportaciones al estudio de la actividad física y el deporte, II Congreso de ciencias de la actividad física y el deporte, Facultat de Ciències de l'Activitat Física i l’Esport, Universidad de Valencia,115-123.

Marco, J. (2003). Psicología, Influencia en el rendimiento deportivo, Madrid, Gymnos,

Moraes, A. (2005). 'La selección de talentos en el deporte de alto rendimiento'. Obtenido, desde: http://www.efdeportes.com/efd91/talento.htm

Parlebas, P. (1997) Problemas teóricos y crisis actual en la Educación Física. Obtenido, desde: http://www.efdeportes.com/efd7/pparl71.htm

Platonov, V. (1995). 'Bases para la preparación a largo plazo de los deportistas de alto nivel'. En Unisport: el deporte hacia el siglo XXI, deportes no 19, Malaga, Instituto andaluz del deporte, 395-403.
Rius i Sant, J. (1995). Formación de jóvenes deportistas, Madrid, Ediciones pedagógicas.

Ruiz Pérez, L. (1999). Rendimiento deportivo, La Mancha, Cuenca, Editorial Universidad de Castilla.

Salmela, J, Regnier, G. (1983). 'A model for sport talent detection'. En Sports: Science periodical on research and technology in sport, Osc., 1 -8.

Sanchez, L. (1998). La detección temprana de talentos deportivos, La mancha, Cuenca, Universidad de Castilla.

Shonborn, R. (1984). 'Problemas relacionados con la selección, búsqueda y evaluación de talentos'. En Simposium F. Europea-Marbella 84, Madrid, Escuela nacional maestría de tenis.

Stojan, S. (1984). 'Búsqueda y selección de talentos'. En Simposium F. Europea-Marbella 84, Madrid, Escuela nacional Maestría de Tenis.

Sáenz-López, P, Giménez, F., Sierra, A., Ibáñez, S., Sánchez, M. (2003). 'Análisis de la formación del jugador experto de baloncesto en España'. Obtenido, desde: http:// www.unex.es/eweb/CIB2007/anteriores/cib2003/ documentos/comunicaciones/CLSaenzLopez.pdf

Terreros, J. (2002). 'Los reconocimientos médicos en el deporte de base como elemento de prevención y mejora de la condición física'. XX seminario aragonés "municipio y deporte", actas, la calidad en el deporte de Base, Zaragoza, Diputación general de Aragón, 121-132.

Torres, J. (1998. 'La detección y selección científica. El modelo de laboratorio de los países del este. Un modelo globalizador para deportes de equipo'. En La detección temprana de talentos deportivos, La mancha, Cuenca, Universidad de Castilla.

Vergara, M. (1999). 'A quienes llamamos niños brillantes'. En Revista Educar hoy, Año 3, Mayo.

Seirul.lo F. (1999). 'Planificación del entrenamiento en la iniciación del futbolista'. En Nazioarteko futbol elkaraldiak, Bizkaia, Editorial Bizkaiko Foru Aldundia, 247-254. 